\title{
Mechanism of carbon partitioning towards starch and triacylglycerol in Chlorella vulgaris under nitrogen stress through whole-transcriptome analysis
}

\begin{abstract}
This study aims to elucidate the mechanism of carbon partitioning towards starch and triacylglycerol (TAG) under nitrogen stress. Whole-transcriptome analysis was performed using RNA sequencing before being validated with qRT-PCR. The strain, Chlorella vulgaris UPSIJRM01, exhibited a two-stage response to nitrogen stress: i) carbohydrate accumulation, plastid protein degradation and amino acid biosynthesis; and ii) lipid accumulation, carbohydrate degradation and DNA damage. In the first stage, the genes responsible for plastid protein degradation (CD4A), amino acid biosynthesis (arg, GLSN, AHCY, win1), carbon fixation (RuBisCO, GapC, fba, TK) and starch synthesis (SS, SBE2) were upregulated. In the second stage, the upregulation of genes responsible for starch degradation (SP), glycolysis (PFO, PK, ACS), fatty acid biosynthesis (FabH, accD) and TAG accumulation (DGAT2) were observed. By contrast, the components of photosystem I (PsbD, PsbC, PsbB), photosystem II (PsaA), cytochrome b6/f (PetA, PetG) and F-type ATPase (beta, alpha) were downregulated. The results suggested that nitrogen stress triggered high starch accumulation before the carbon was partitioned towards triacylglycerol (TAG) for long-term energy storage by two different pathways: chloroplastic TAG synthesis and glycerolipid metabolism. The starch build-up functions served as a rapid response to nitrogen stress, whereas subsequent lipid accumulation benefited the long-term storage of energy. This research provides in-depth understanding of the metabolic changes triggered by nitrogen stress.
\end{abstract}

Keyword: Chlorella vulgaris; Gene expression; Nitrogen stress; RNA sequencing; Carbon partitioning mechanism 